\title{
REV. DANIEL LANE AND HIS KEOSAUQUA ACADEMY
}

\section{BY J. W. CHENEY}

On his mother's side Daniel Lane was a descendant of John and Priscilla Alden, members of the Mayflower band of Pilgrims. He was born in Leeds, Kennebec County, Maine, March 10, 1813. Hís father kept a country store, and, evidently, was in very moderate financial circumstances. Daniel was the only child of this mother, who must have died when he was a mere baby, for when he was only four years old his fatheralso died, leaving to the little Daniel a step-mother and a half-sister. He then found a home with his own mother's brother, a Mr. Brett; not that his step-mother lacked in affection for him, but because she was left with insufficient means to properly support herself and the two children. She lived until after Mr. Lane was thirty years old, and he always spokè very highly of her.

Hon. A. J. MaCrary says of Mr. Lane, "He was truly the friend of youth, yet you could not think of him as ever having any youth." But in everry case the broy precedes the man. The little I know of Daniel Lane, the boy, I learned. from the Valentine brothers, Lowell and John, who followed Mr. Lane to Keosauqua, Iowa, and whose mother was a consin - of Mr. Lane's mother. Lowell Valentine was superintendent of the Congregational Sunday School in Keosauqua when I was a boy, and I recall his telling a very interesting story of the struggles and triumphs of a poor, orphan boy, closing with the impressive declaration, "And that boy was Daniel Lane." At the time we had no difficulty in thinking that Mr. Lane might have been such a boy. But John Valentine, who now lives in Denver, Colorado, writes me something which may astonish those who only knew the man, Rev. Daniel Lane, and are not able to " think of him: as ever having any youth." $\mathrm{Mr}$. Valentine says, "I can tell you an incident of his boyhood, which not only illustrates his desire to excel in everything, 
but also shows the power of religion to change one's moral nature. And I have this from his own lips. Several boys, of whom Daniel was one, were playing together, when some of them became very profane. Daniel so far excelled the others that one of them, at least, was greatly shocked and exclaimed, 'Now Dan! quit that!' And Daniel was so surprised and deeply moved by the rebuke that he did quit, then and there, and soon afterward became a follower of the Jesus whose name he had used so lightly." That indeed was the turning. point in his career, and the real key to his future character and useful life.

As nearly as I can learn he was about sixteen years old when, after much reflection and study of the Bible, he came out openly on the Lord's side and united with the Congregational church. He fitted himself for college in the Brighton Academy. While doing so his health became very poor, he was thouglit to have consumption, and asked his physician whether he would better go on with his studies. The reply was, "Oh yes, but you will not live beyond your second year in college." He did go on, and not only passed "the dead line" safely, but graduated from Bowdoin College in: 1838, by which time he was twenty-five years old. In the meantime he had taught school in several places, among which was the village of Freeport, not far from the city of Portland, Maine. There he became acquainted with the family of David Staples, a sea captain, whose daughter Elizabeth was destined to be his devoted wife and efficient helper throughout his career in Iowa.

Immediately after graduating from college he became the teacher of English and modern languages in North Yarmouth Academy. At this writing, 1915, there is living in Jowa City a Mrs. Saunders, who was then a student in that academy, but probably in the primary department, as she was only nine or ten years old, and only remembers that Mr. Lane was a tall, slender, fine looking man, and very highly esteemed by the whole community as a man and teacher. After teaching two consecutive years in Yarmouth, he entered Andover Theological Seminary, took its three-year course of study, and graduated therefrom in 1843, at the age of thirty years. 
While in the seminary Mr. Lane, because of his riper years and strong personality, became a leater among the students, especially those of his own class. But in the summer of 1843 , near the close of his second year at the seminary, he was in very poor health, and it is he to whom the author of "The lowa Band" refers in relating what occurred one evening that summer at the usuat devotional exercises of the faculty and students: "Among them sat one, pale and emaciated by continued illness, and of whom his friends began to whisper, 'Unless relieved soon we fear' he will never he well, even if he lives.' They might have spared a portion of their anxiety had they known the nature of his disease, which was dyspepsia, and that not of a chronic form." Mr. Tane eame to that service greatly cast down by the combined effects of disease and hard study. During the service he deeply pondered his condition and prospects, and had about concluded that he must abandon his long-cherished plan of becoming a New England minister, for the reason that such a life would aggravate his disease, cripple his eneroies, and shorten his days. At that moment there came to him the thought that the quite different life of a missionary in the west might counteract his disease. To go west would require great self-denial, but there might be great compensation, chiefly of a spiritual character. These thoughts, with others, passed before him with the swiftness of a vision, and had for a time the effects of a vision. All things else were shut out. The chapter, the hymn, the singing were all unheard. In the general movement he rose for prayer, but not to join in the petitions offered. The spell was upon him, and he seemed to stand alone before God. He went out that evening not as he came in. Henceforth his prayer was "May I be found in the right place, doing the right work! Prepare me for it, and make me willing to enter upon it!' 'The result was that he definitely decided to become a western missionary. He soon found that a classmate from the west expected to return and labor in that region. And these two so successfully promoted the scheme that ten others of their class joined them. The twelve prospective home missionaries were Daniel Lane, Harvey Adams, Erastus Ripley, Horace Hutchinson, Alden B. Robbins, William Salter, Edwin B. Turner, Benjamin A. 
Spaulding, William Hammond, James J. Hill, Ebenezer Alden and Ephriam Adams.

These kindred spirits then proposed to hold prayer meetings, to further foster their remarkable friendship and unity of purpose. But no two of them roomed together, and the question arose as to when they might privately assemble. One of their number happened to be the seminary librarian; so they decided to meet in the library room, although the seminary rules forbade lights in that rom: but they overcame that difficulty by meeting there on 'Tuesday evenings and praying in the dark. And in after years, though widely separated in the mission field; those devoted men observed Tuesday evening as the set time to secretly pray for each other. Before graduating from the seminary the twelve had chosen. Iowa territory as the field of their missionary labors. They therefore became known as "The Iowa Band."

After seven years of acquaintance, courtship and betrothal, Daniel Lane and Elizabeth Staples were married, September 9, 1843, which was soon after he graduated from the seminary, and a few weeks before "The Band" was to start 'west. One of the members, William Hammond, decided not to go at all, "for fear of the western climate," and two more, Erastus Ripley and J. J. Hill, were temporarily detained, and came on the following year. Nine of "The Band," two of them, Mr. Lane and Mr. Robbins, with wives, started on the long journey, Oct. 4, 1843. The first stage was by train to Buffalo, then the western terminus of the railroad, thence by a lake steamer to Chicago. It is worth noting and will amuse present day Iowans, that during a brief landing at Milwankee they were met by Rev. Peet, the Wisconsin agent for the American Home Missionary Society, which was financing "The Band," but he discouraged their going on to their destination by saying "Iowa will never amount to much, as it has only a narrow strip of good land along the Mississippi river, beyond nwhich is the Great Amer. ican Desert." The only excuse for such a statement was igrorance of the character of the unsettled portion of Iowa at, that time, when it was understood that "the. settled portion of the territory was a belt of land on the west bank of the Miss- 
issippi, 200 miles long and 40 wide, with a population of something over fifty thousand." From Chicago, by chance con. veyances, mostly open farm wagons, the missionaries came through what was to them, "a new and wonderful country," and were much surprised to get good meals by the way for" a "bit," $121 / 2$ cents, and night lodging for 25 cents. Through out the whole trip they refrained from travcling on Sunday and, after about seventeen day of actual travel, they arrived at Denmark, Lee County; Iowa, October 25, 1843.

But they were not the first Congregational missionaries to come, to Iowa. 'The same missionary society had sent Rev. Asa Turner to Quincy, Illinois, in 1830. In 1836 he made an exploring tour to the Black Hawk purchase, and found a colony of religious New England people settled in the Denmark locality. In 1838 those Denmark people invited him to become their pastor; he accepted the call, and sustained that relation to them for thirty years. However, during the first six years of that period he gave half his time as agent for the "American Fome Missionary Society in the territory of Iowa. Fourteen Congregational churehes had been organized by the time the "Iowa Band' came, and some eight Congregational ministers had reached the Territory," so said Dr. Magoun at the dedication of a new Congregational church in Keosaukua in 1888," and Dr. Salter one of 'The Band,' says, 'It was a letter from Asa 'Turner, under God, more than any other singte influence, which led us to choose Iowa as our field of labor,' therefore, with or without the consent of my Congregational friends, I may say that Asa Turner was a sort of Bishop of Iowa, and Denmark the headquarters of his diocese; which accounts for "The Iowa Band' coming to Denmark in a body."

Dr. Salter further relates that, after arriving in Iowa, "the next Sunday I spent at Keosanqua, on the Des Moines river, and preached in a blacksmith shop." A Mr. Hadden attended or followed him back to Denmark where, on the following Sunday, November 5th, Mr. Isane and six others were to be ordained before the members of "The Band" dispersed to their appointed fields of labor. The method of assignnent to those fields is thus deseribed in the little book entitled "The Iowa Band", the nine members having assembler in the pastor's 
study for that purpose: Then Fathers Turner and Gaylord, who had explored the field, came in, map in hand, described their tour, the places visited, and then retired. Now, by free suggestion and mutual consent, the assignment began. Brother Hutchinson, for peculiar reasons, as was well known, was inclined to Burlington, and $\mathrm{H}$. Adams to Farmington; and none were disposed to object. Those having wives, it was said, ought to be provided with places as comfortable as any in the territory. A minister-seeking man" "from Keosauqua had claimed Brother Lane as the one of his choice. His promises were fair, and he was gratified. Then Bloomington, since called Muscatine, a smart town of 400 inhabitants, was ceded to Brother Robbins, and thus the wives were provided for." And thus, incidentally, was shown the rank which Keosauqua held among her sister towns in 1843. 'The Savior's injunction was "judge not according to appearances; judge righteous judgment." For lack of time and opportunity Mr. Hadden lad to "judge according to appearances" when he chose Mr. juane but, fortunately, it proved to be a "righteous judgment" also, and has been endorsed as such by Keosuaqua people unto this day.

Mr. Lane was nearly thirty-one years old on November 12, 1843, when he preached his first sermon in Keosauqua, and stood face to face with the great work the had chosen, and for which God had chosen him. Many precious years had been spent in preparation for it, not willingly but necessarily. He had not inherited a robust body; physical weakness always, and real illness often, hindered study. And a degree of poverty frequently drove him from the halls of learning, and compelled long periods of manual labor or teaching, in order to replenish his normally slender and often empty purse. His eager spirit chafed against the enforced delay, which after all was not without its compensations, for the protracted struggle was a discipline which resulted in the development of patience, courage, perseverance, self-reliance-all those moral qualities, indeed, which characterized him afterward and contribnted so much to his popularity and usefulness.

As a matter of economy, if not of necessity, the Lanes had sent their few household effects by water down the Atlantic Mr. Hadden. 
coast, across the Gulf of Mexico, and up the Mississippi to Burlington, whence they must come to Keosuaqua by wagon; and until they arrived $\mathrm{Mr}$. and Mrs. Lane boarded with Mr. Hadden's family. How few were those household effects is shown by the following excerpt from the diary of Rev. H. Adams, of Farmington, who, the next summer, visited the most of his brother ministers at their homes, beginning at Keosanqua: "July 16, 1844. Here are Brother Lane and his wife in their little home of two rooms. They have a chair or two now and a table, but they say they set up housekeeping without either, using old boxes instead." He then goes on to say "They have a churoh of a few members, organized as Presbyterians, but its members are not all of that way of thinking. Brother liane is coming to be very decided that Congregationalism is the true Bible way, really quite conscientious about it. A majority are with him. How things will turn out, can't tell." How "few members" com. posed that church, and how eager was "the majority" who were "with Brother Lane" on the denominational question, appears from the fact that, when he did organize a Congregational Church about four months after Rev. A'dams' visit, and a little more than a year after Mr. Lane begani his labors here, he did it 'with only five members, viz., Moses Root and wife, Comfort Barnes and wife, and Mrs. Lane. Moreover, Mrs. Lane was the only memiber who lived in town; the others lived two and four miles out. Mr. Hadden, the Chief instrument in bringing Mr. Lane to Keosauqua, must have been a Presbyterian "after the strictest of his sect," for he did not then join the Congregational church, nor did he afterward during the few years he remained in town.

When Mr. Lane had been in Iowa about two years, the condition of his health required an extended vacation and a change of climate, but did not keep him from doing good when and where he had opportunity: Of that vacation $\mathrm{Mr}$. John Valentine writes me, "The winter of 1845-6 Mr. Lane spent in Maine, on account of illness from malaria, and made his home with my brother, Lowell. During that winter he' preached to our people there; and it was then under his preaching, that I. was converted." 


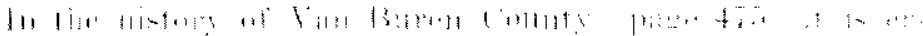

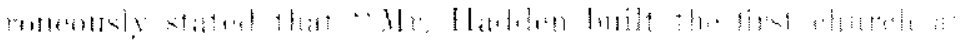

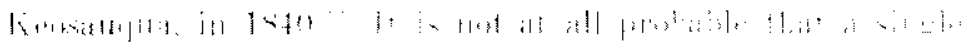

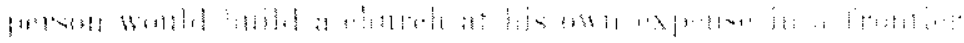

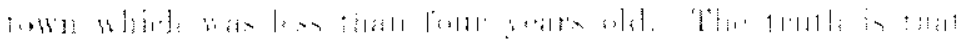

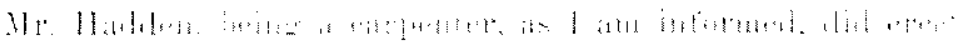

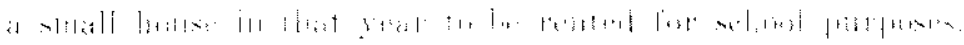

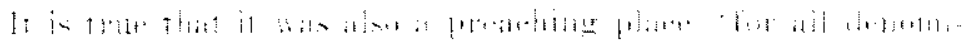

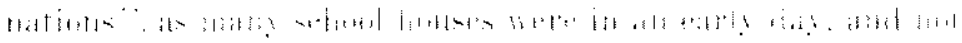

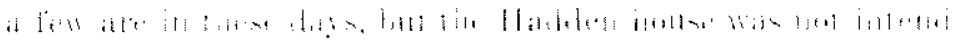

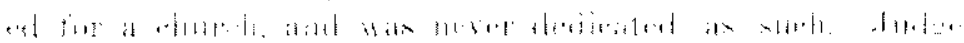

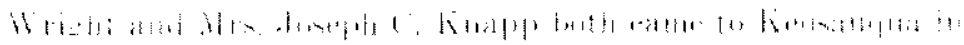

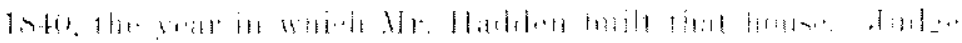

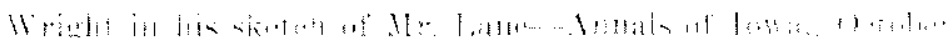

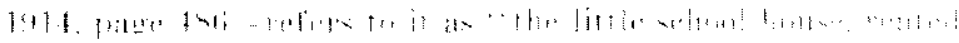

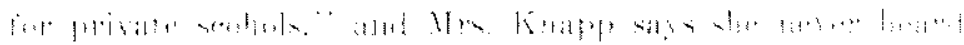

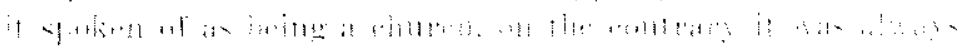

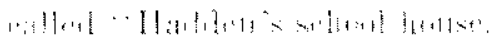

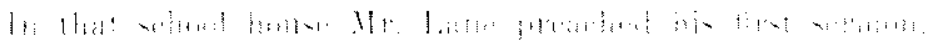

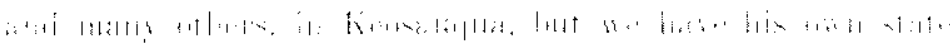

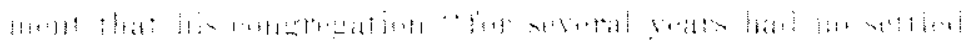

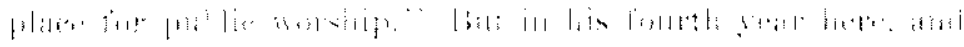

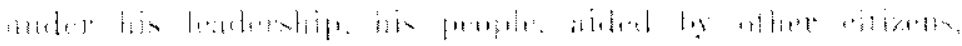

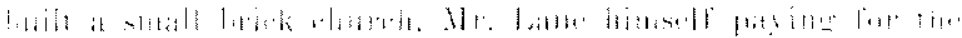

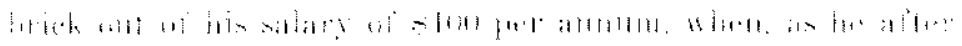

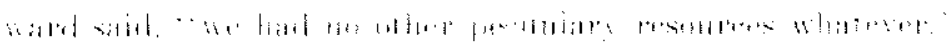

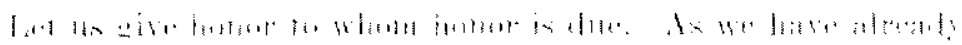

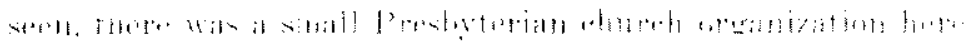

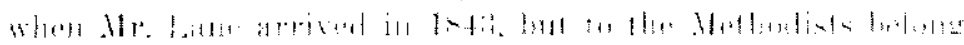

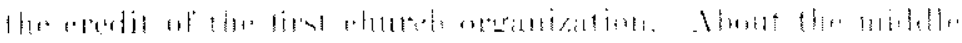

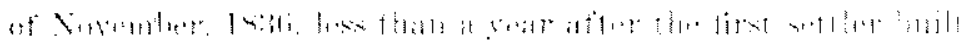

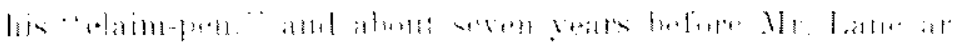

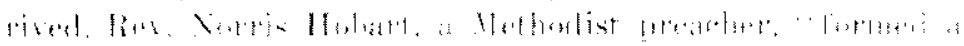

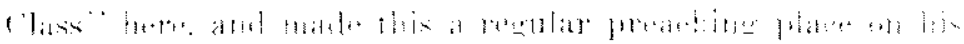

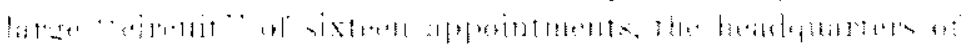

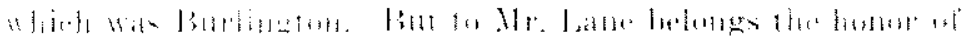

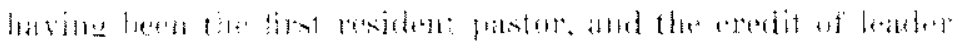

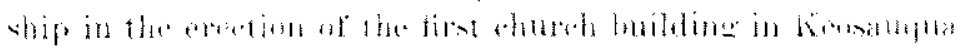

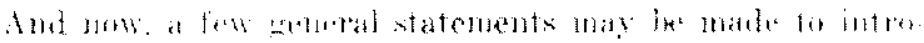

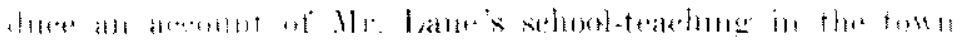




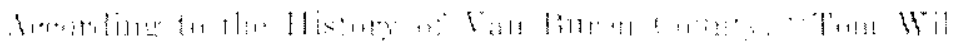

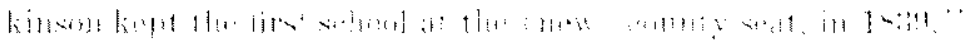

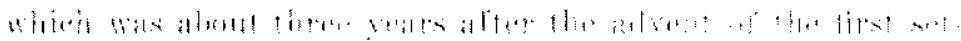

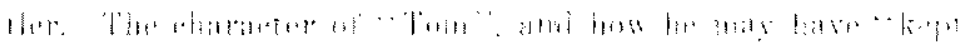

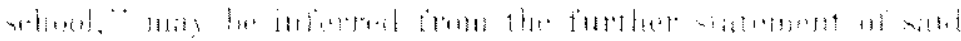

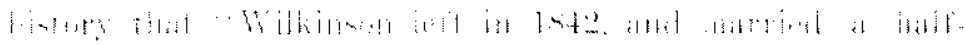

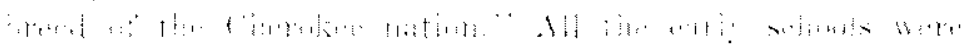

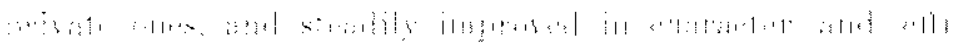

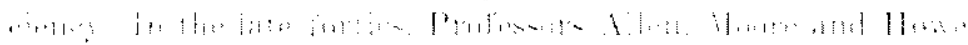

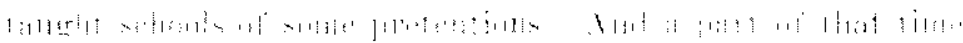

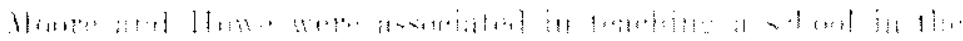

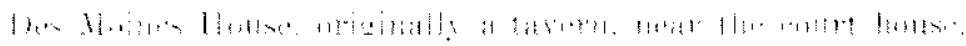

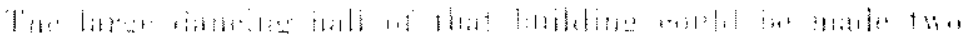

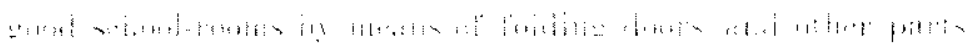

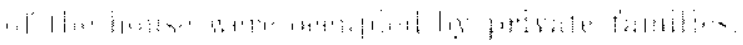

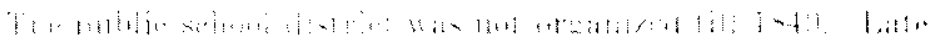

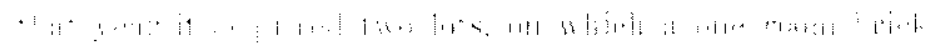

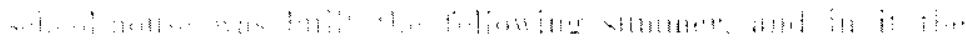

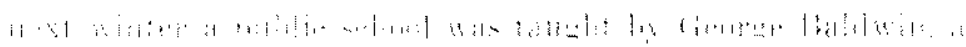

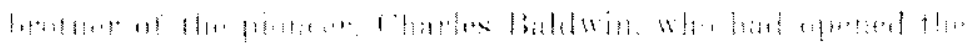

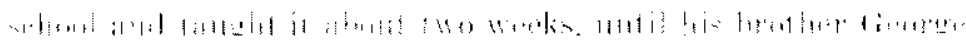

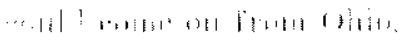

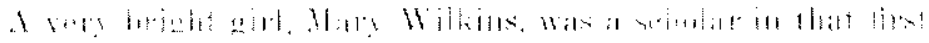

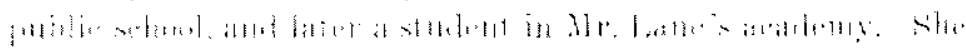

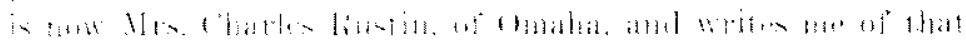

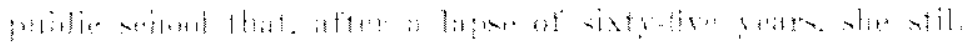

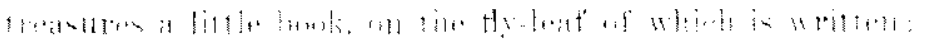

To Misk Mary E. Wilkins:

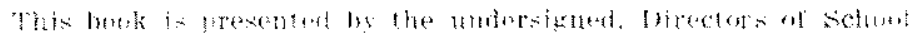

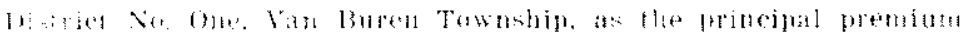

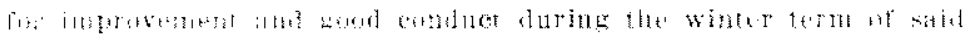

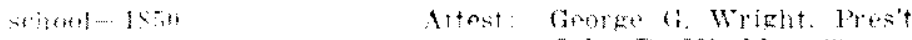

John D. Niteher, Tretis.

John H. Stine, see.

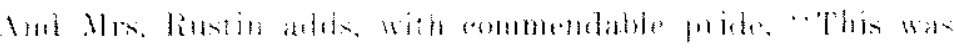

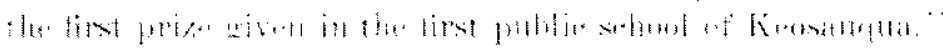

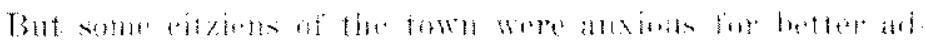

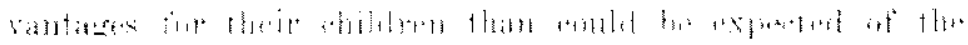

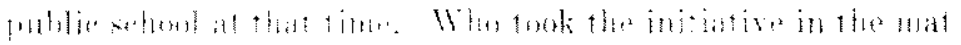

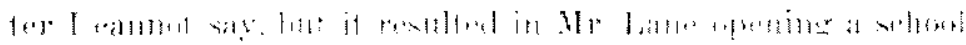




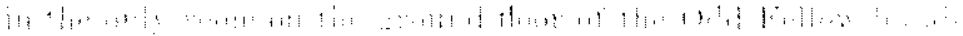

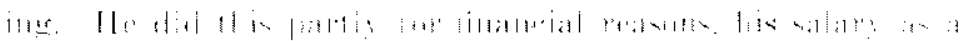

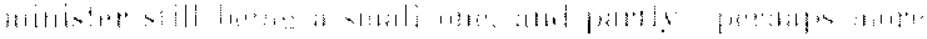

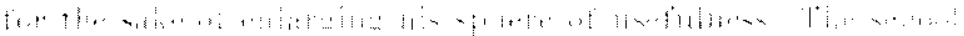

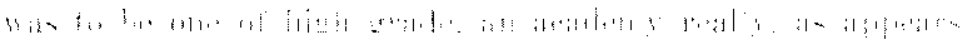

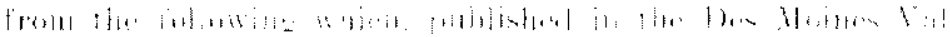

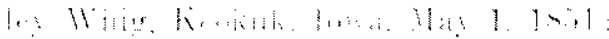

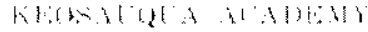

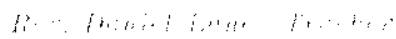

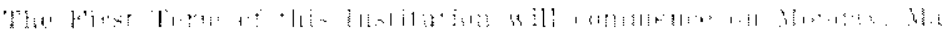

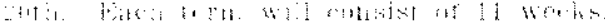

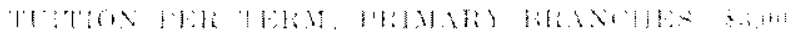

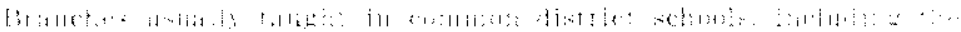

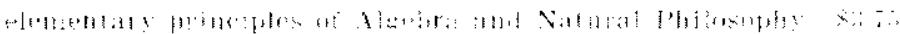

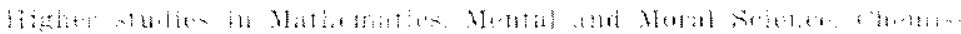

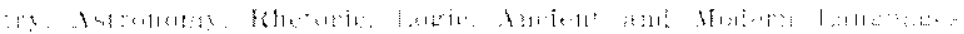

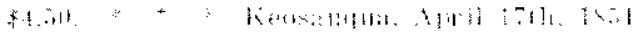

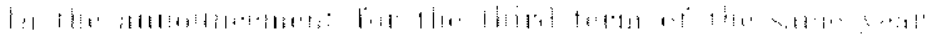

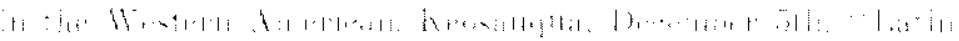

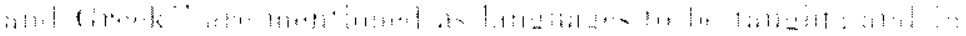

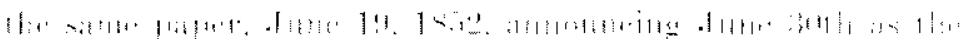

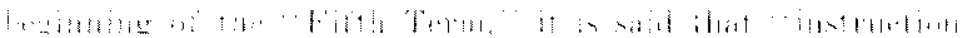

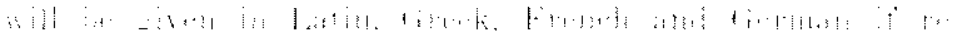

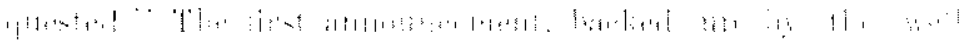

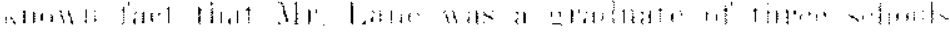

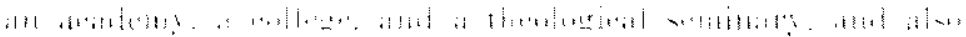

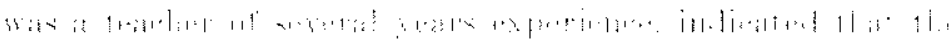

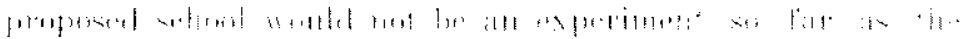

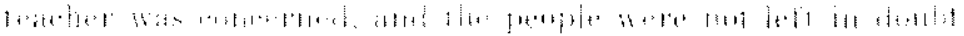

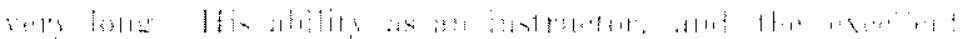
tan?al at

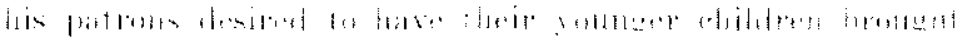

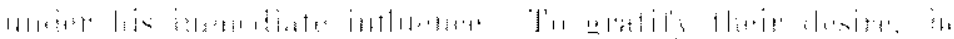

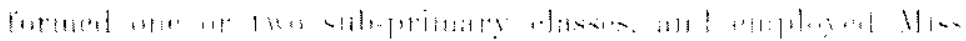

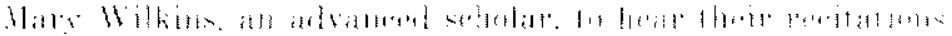

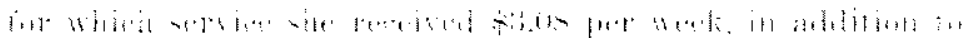
1)+2 +

The now

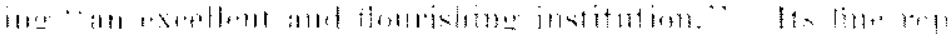




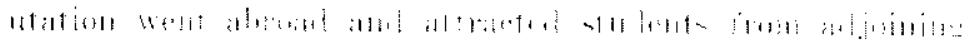

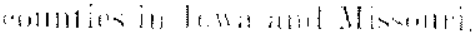

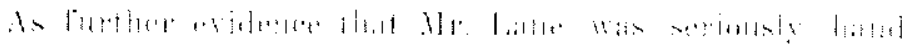

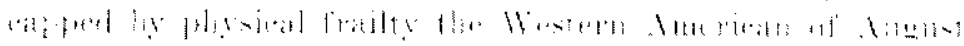

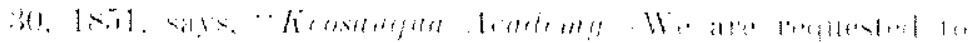

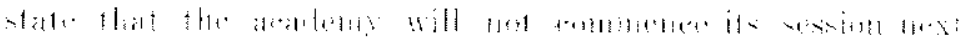

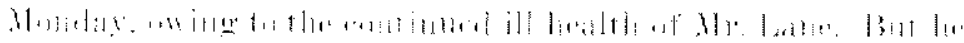

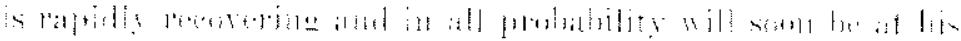

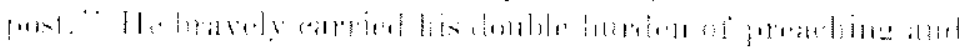

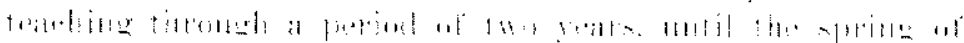

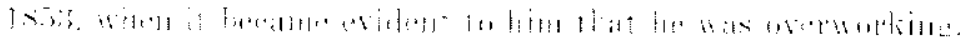

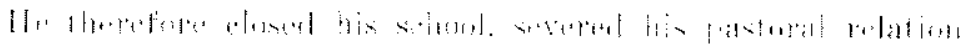

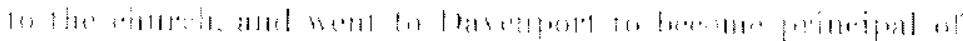

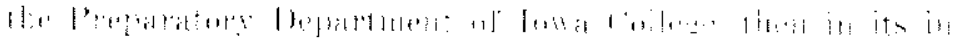

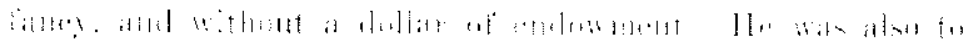

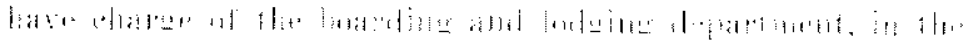

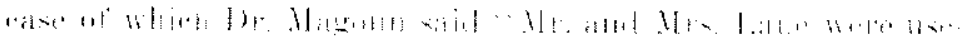

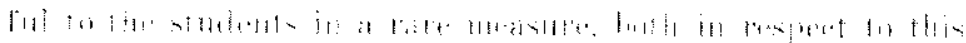

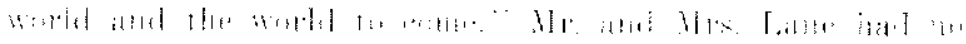

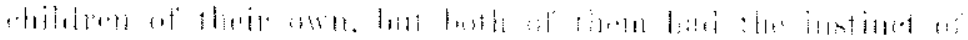

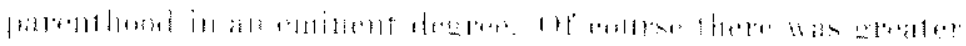

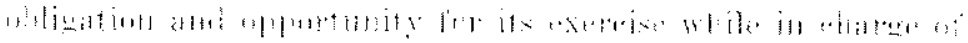

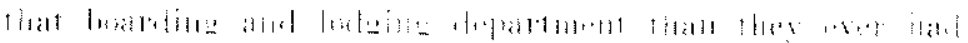

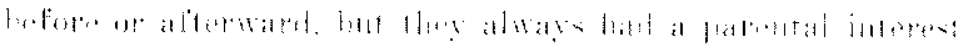

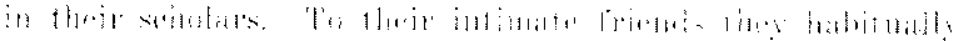

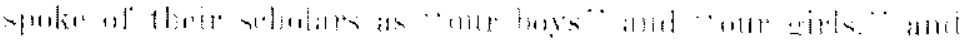

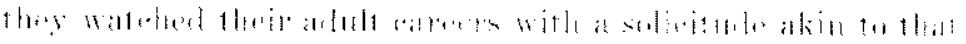

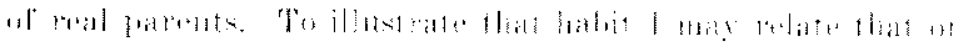

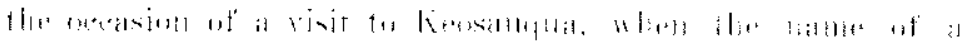

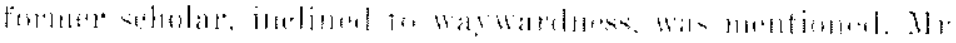

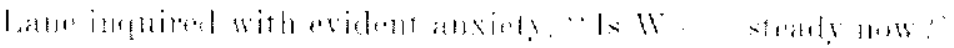

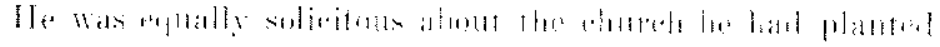

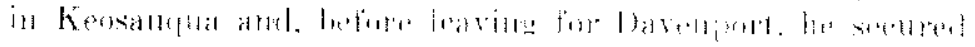

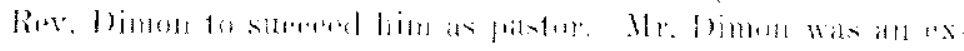

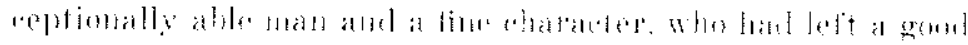

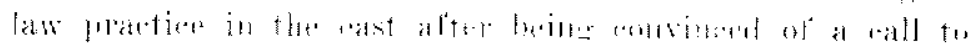

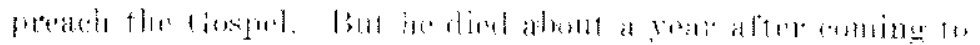

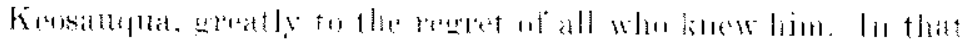

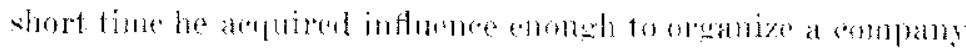

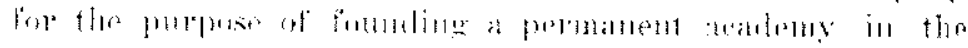




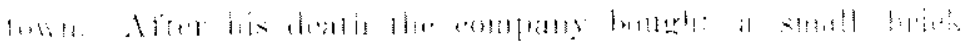

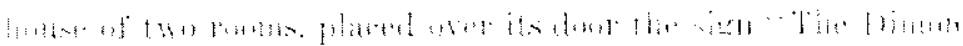

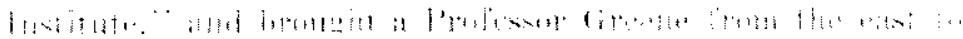

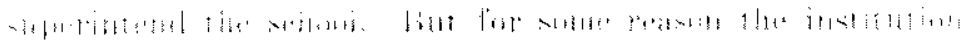
a

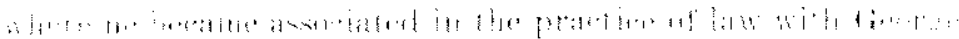

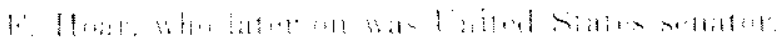

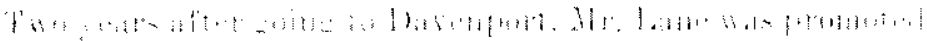

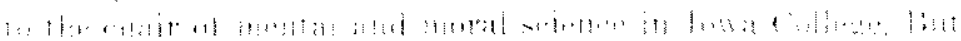

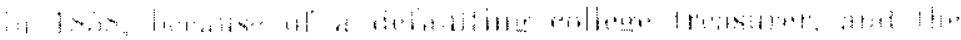

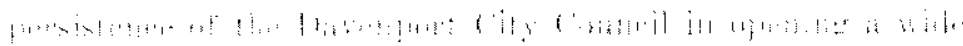

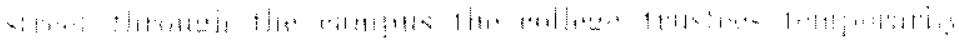

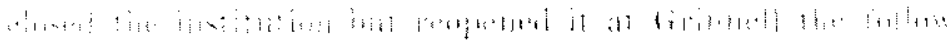
i... $\cdots+\cdots$

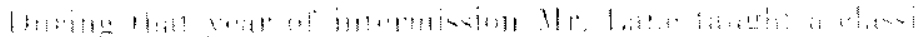

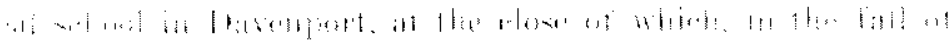
I r. If

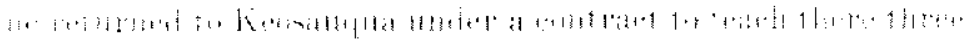

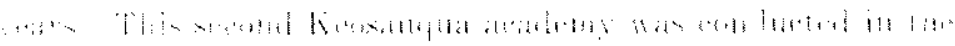

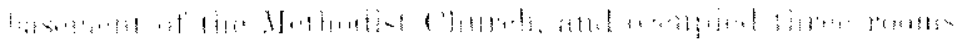

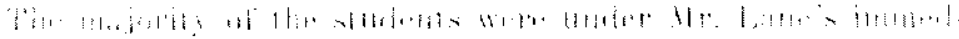

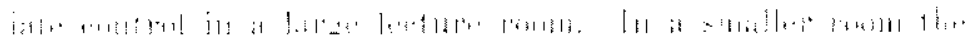

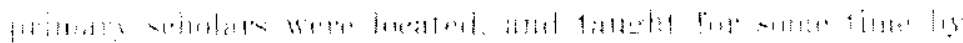

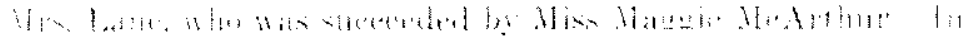

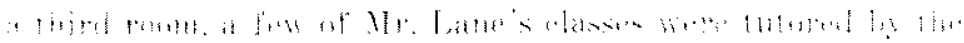

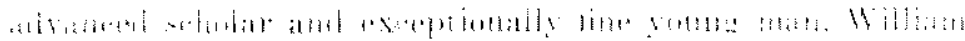

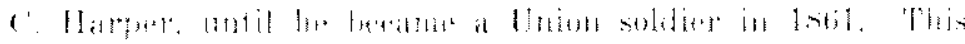

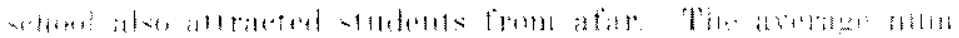

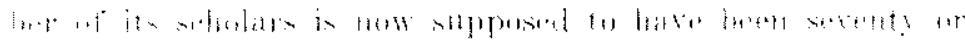

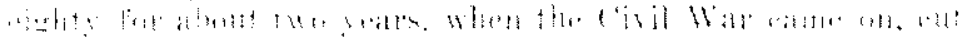

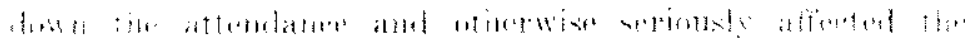

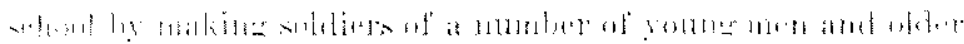

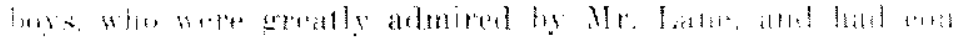

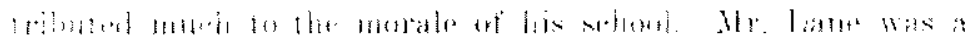

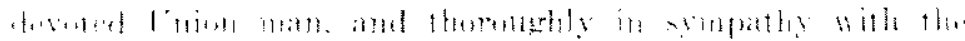

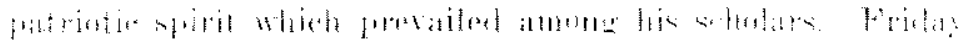

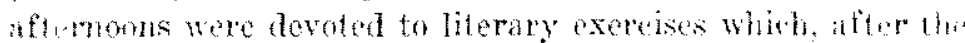

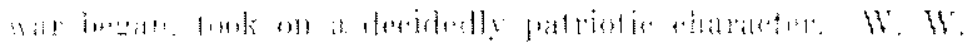

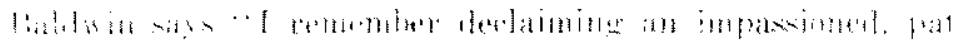

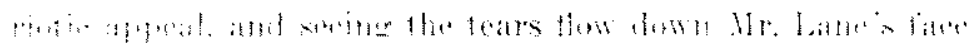




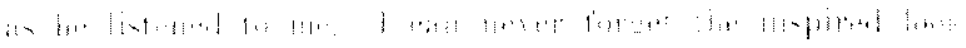

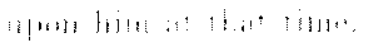

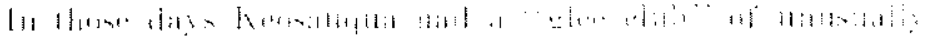

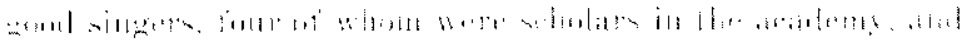

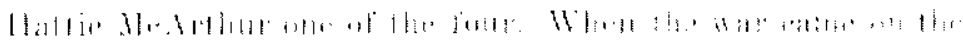

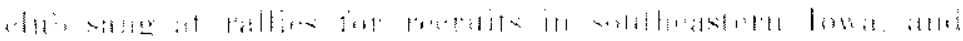

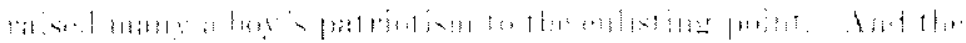

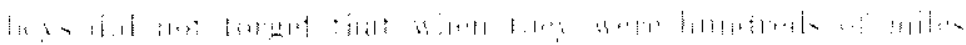

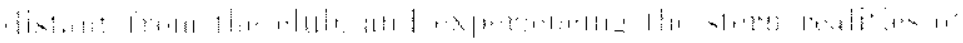

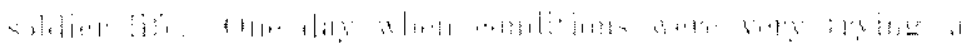

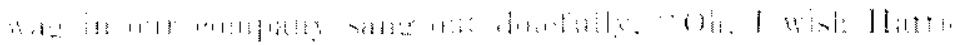

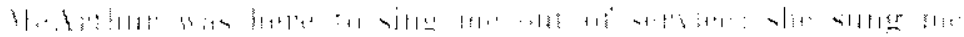
Hil: il:

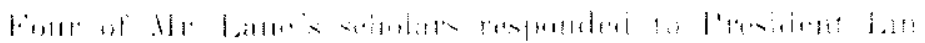

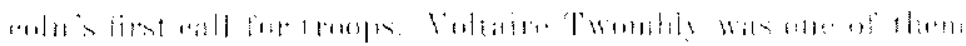

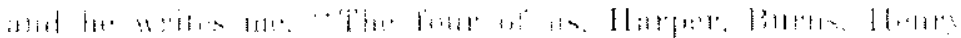

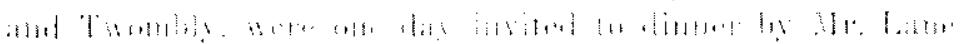

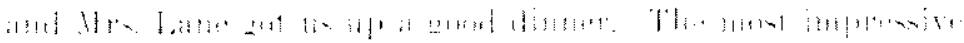

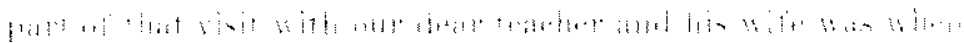
in! all

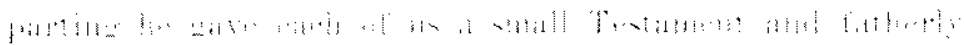

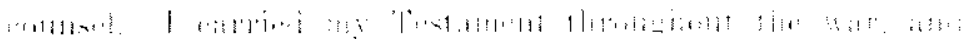

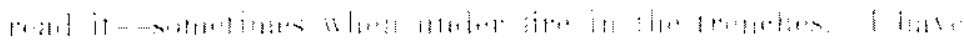

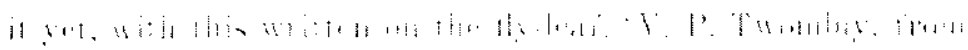

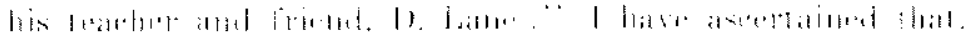

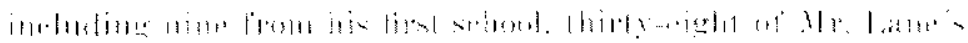

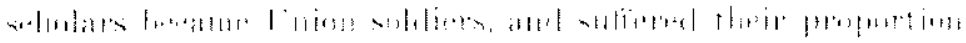

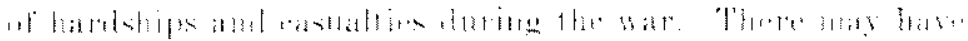

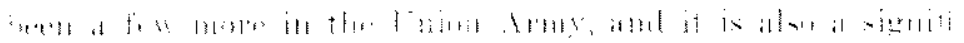
.all

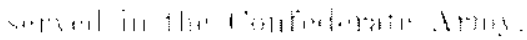

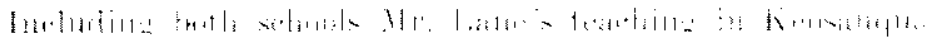

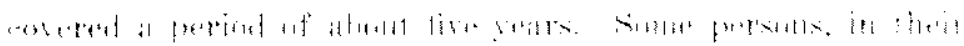

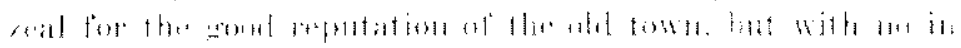

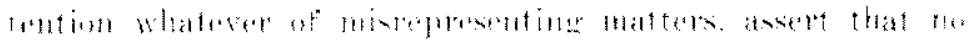

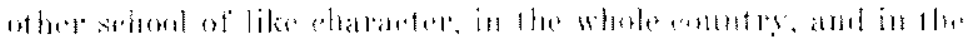

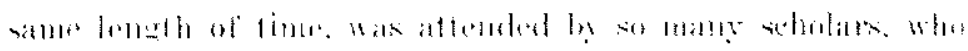

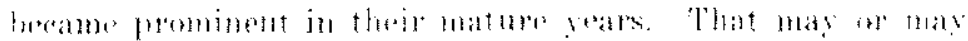

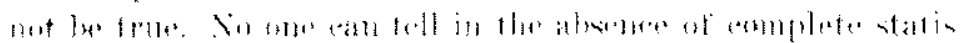


tics from all such schools, and it is safe to say that no such statisties were ever compiled, and distributed, therefore the assertion may be made only as an opinion, not as a known fact. Moreover, the makers of the foregoing statement erroneously, but of course honestly, swell their mental list of Mr. Lane's scholars who became prominent men, by including in it the namess of George W. MeCrary, H. C. Caldwell, and a few others, of less prominence, none of whom ever went to school to Mr. Lane. In the Annals of Towa, Oetober, 1914, there appeared a brief character sketch of Mr. Lane by Judge Wright. In that sketch the Judge does not assert the superiority of Mr. Lane's school over other schools in the production of prominent men, but he restricts the field of comparison, and adroitly shifts the burden of proof upon any one who might deny it. He says, "Find if you can another instance in this western world, in the early days, of a small private school sending ont so many men of whom the instructor, the state and nation even, may feel so justly proud." This challenge comes after naming twelve prominent men-from memory - who had been scholars in Mr. Lane's school, Judge Caldwell among them. I will not attempt to take up the challenge, for $I$ do not contend for the superiority of any other school, but the Judge is mistaken in naming Caldwell as a student in the Lane Academy. This may seem strange-and it is-in view of the facts that Caldwell studied law in the office of Knapp and Wright, and was junior member of the firm of Knapp, Wright \& Caldwell from the time he was admitted to the bar in 1851, until he enlisted as major in the Third Iowa Cavalry in 1861, a period of about ten years.

Now Judge Wright's reminiscent articles are very fine. They are illuminating, intensely interesting, and, in a general way, are faithful portrayals of pioneer characters and events. But they were written exclusively from memory, or nearly so; and it is well known that memory is not perfectly reliable as to the details of forty or fifty years "Lang Syne." In other articles I have found Judge Wright in error as to some details. And in the article now under consideration there are two errors besides the one concerning Caldwell. The first one states that Mr. Lane settled in Keosauqua in 1842 instead of 1843, and the second says "forty years later he returned to his first 
home in Maine, and died within the year," whereas he lived over seven years after returning to Maine. It is thought by some that George W. McCrary attended school in Keosauqua and it is known that Caldwell went to school to Professors Allen, Moore and Howe; but I have reliable information that neither of them, nor a few others included with them, ever were students in Mr. Lane's schools. Mrs. Rustin, Winifield Mayne and others who were students in the first school are quite positive that those persons did not attend it, and Mrs. Knapp confirms their statement.

Mrs. Rustin explains that as the Knapp, Wright \& Caldwell law office was just across the street firom the academy school room, Caldwell would often come over during intermission periods and join the older scholars in their games.

Caldwell had very little schooling, but he was one of those percocious youths who had a faculty for obsorbing knowledge, and made good in after life. He began studying law with Knapp \&. Wright at the age of fifteen and was only nineteen when he was admitted to the bar in 1851, the very year in which Mr. Lane opened his school in the Odd Fellow building. What more likely then than that the boy of nineteen. or twenty should still have a zest for play and often engage in it, when suitable comrades were hard at it so near him, especially when his sedentary occupation made erereise and recreation necessary. Judge Wright saw those youngsters at play hundreds of times; they made a bright and enduring picture in his mind. Forty years later as he sat writing his tribute to Mr. Lane memory" bronght out that picture labeled "Mr. Juane's students at play" and in it was Caldwell, one of the most enthusiastic players; so memory played the honest Judge a trick, and beguiled him into thinking that Caldwell was really a student in that academy. Finally, I. have a list of the students in that school, given by Mr. Lane himself to Thomas S. Wright, the Judge's son, for uss in an address made in Keosauqua in 1888, and the names of George W. McCrary and H. C. Caldwell are not in that list. It is true that Mr. Lane made that list from memory, closing with the remark "And perhaps two or three others, whose names are not recalled by their old teacher." But it is incredible that he should have forgotton two such men as MeCrary and Cald- 


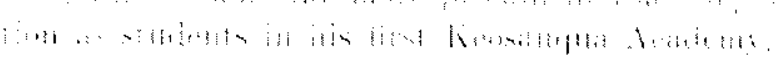

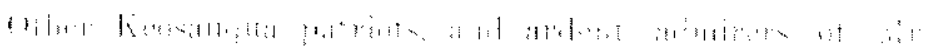

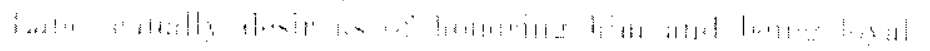

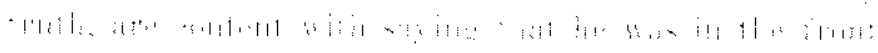

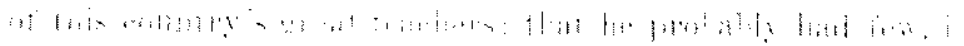

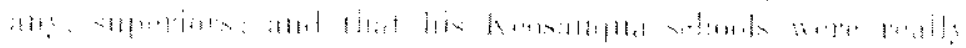

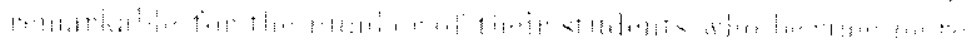

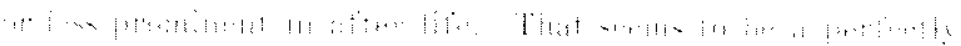

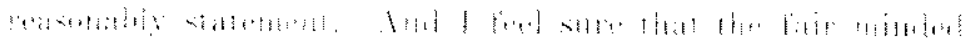

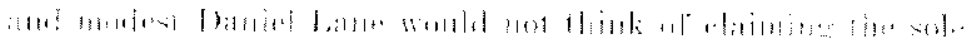

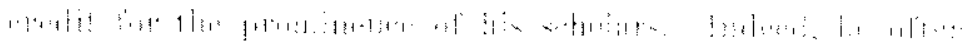
and a

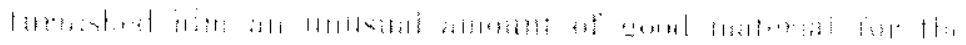

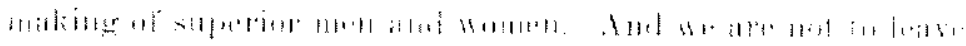

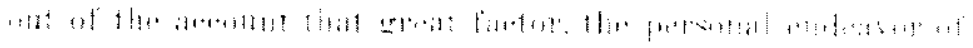

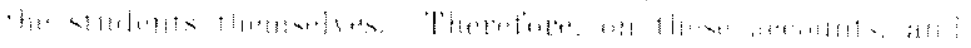

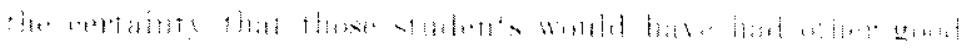

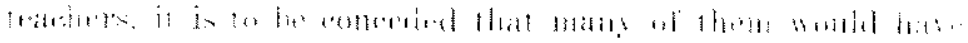

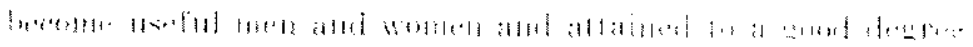

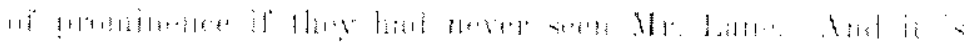

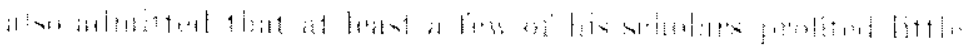

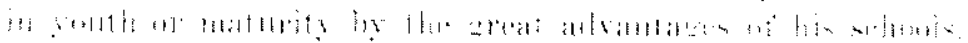

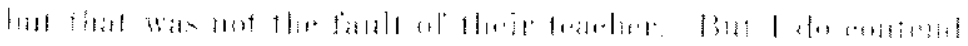

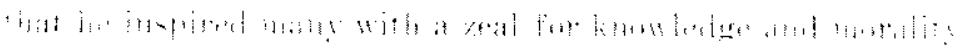

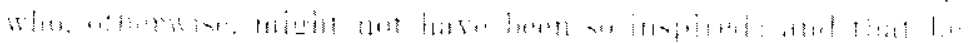

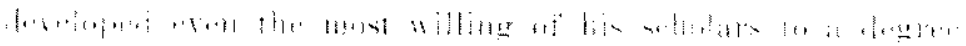

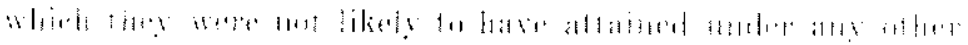

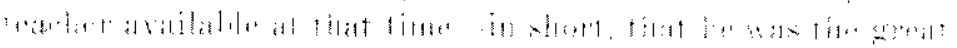

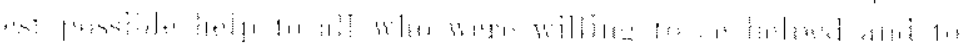

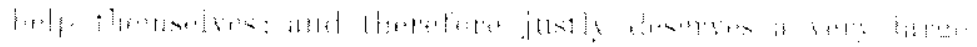

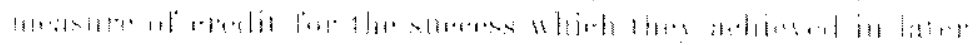
lilin.

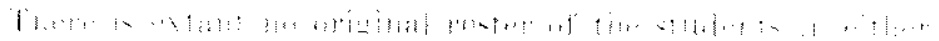

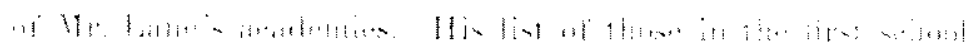

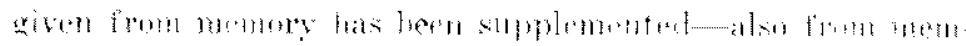

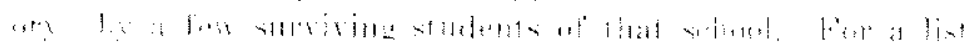

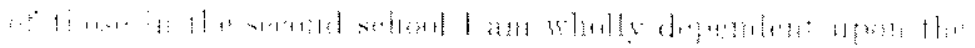

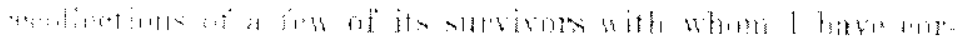

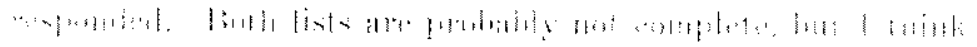


a

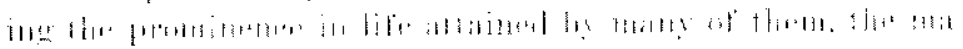

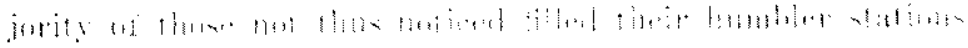

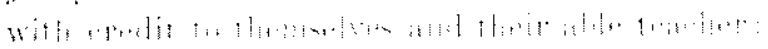

\section{FIRST SAHO(t)}

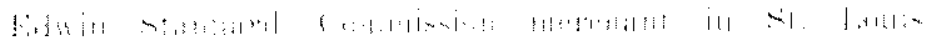

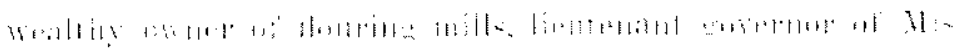

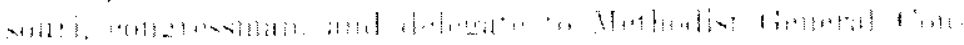

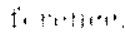

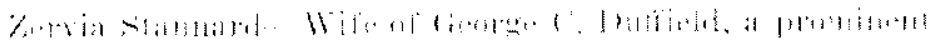

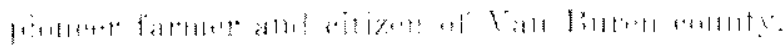

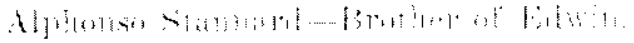

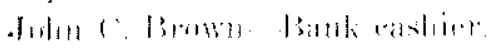

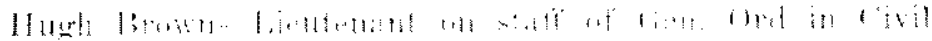

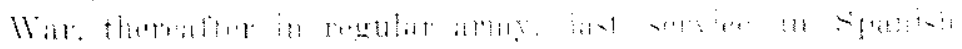

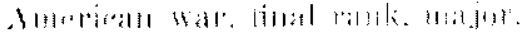

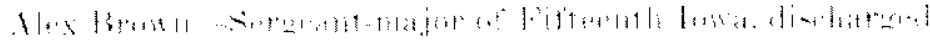

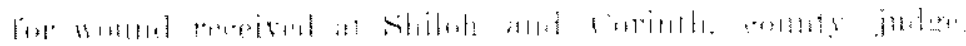

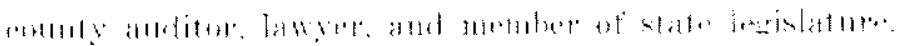

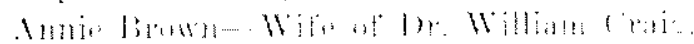

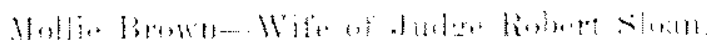

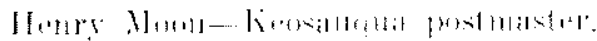

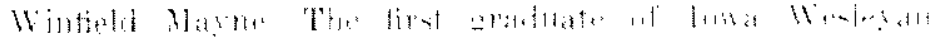

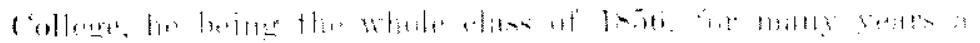

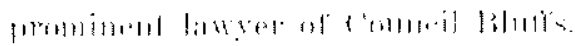

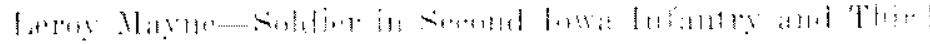

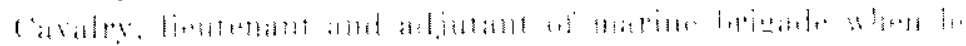
ilind in lsin?

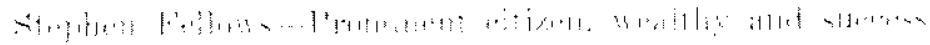
till finlumen.

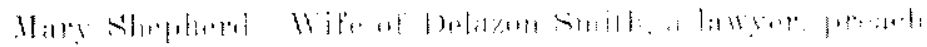

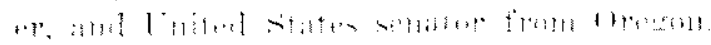

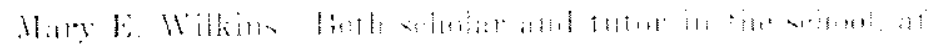

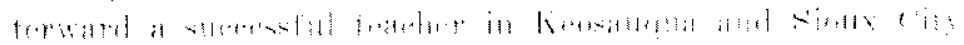

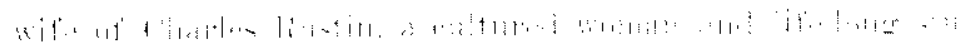
atiol.

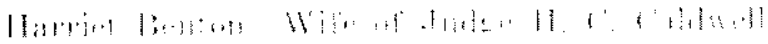

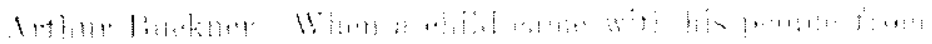

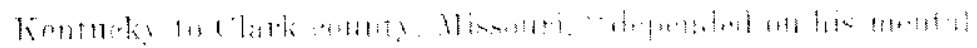


quickness rather than on close application to study, mischievous in season and out of season, and the only scholar I ever saw Mr. Lane out of patience with" says Mrs. Rustin. He became a physician and eminent surgeon. The Confederate Gen. Buckner was his great uncle. Arthur was loyal and served as a surgeon in the Union Army.

Aurelia Julien-Wife of Mraj. H. C. McArthur, civil war veteran.

Jane Bell-Sister of Col. Frank Bell.

Margaret Leach-Daughter of Gen. Leach. try.

Isaac Thatcher-Captain of Company K; Forty-fifth Infan-

Amos Thatcher-Sergeant-major Fifteenth Iowa.

Jacob St. John-Lawyer in Des Moines.

Vina Baldwin- $\rightarrow$ Sister of Charles Baldwin.

Ellen Manguin-Wife of Winfield Mayne.

George Swain-Lieutenant in Seventh Cavalry.

Aurelia or "Milly" Williams-Wife of Mr. Schramm, a prominent Burlington merchant.

Volney Smith-Son of Delazon Smith, was cadet a while at West Point, suposed to have been a soldier in the Civil War, and known to have been prominent in Arkansas polities.

Lizzie Brown, Mary Ann Brown, Nancy Brown, Elizabeth Burns, Mary Burns, Elizabeth Cameron, Cornelia and Mary Chittenden, Ellen Claflin, James Coleman, Samuel Dook, Devin, Davis Leonard, William Fellows, William Fosnot, Sarah and Amanda Hartzell, Victoria Julien, Luther and Mary Kreigh, Henry Mathias, Philander and Carrie Mayne, Sarah Jane, Elizalbeth and Angeline Miller, Jackson and Zarvia Miller, David Miller, Mary Moore, Francis Montonye, Martha Selby, Felissa Stannard, Joanna Steele, Carlisle and Sarah St. John, Louisa and Sarah Tolman, Charlotte and Russell Tylee, Adaline and Amanda Walker, Boylston Wilson, Emily Webster and William Wallace Brown.

\section{SECOND ACADEMY}

The first three names on this list are persons who were also in the first school, but in the primary class.

Charles W. Shepherd-Served three years in Third Iowa Cavalry, then till close of Civil war as a lieutenant in a col- 
ored regiment, was a Methodist minister nearly thirty years, and died when treasurer of Van Buren county.

Thomas S. Wright-Son of Judge George G. Wright, was adjutant in Third Cavalry, prisoner of war for a short time, lawyer of prominence, and was attorney for the C. R. I. \& P. Ry. Co. when he died in New York City as the result of an accident, age about forty-nine years.

V. P. Twombly-Excelled in mathematics while a student, enlisted spring of 1861 in the Second Iowa Infantry at the age of nineteen, was slightly wounded at F't. Donelson and was the last of the color guands on his feet when he carried the colors over the Confederate works, was promoted from grade to grade until made captain, was severly wounded at Corinth, served over four years. After the war was treasurer of Van Buren county four years, treasurer of the state of Towa six years, and president of the Home Savings Bank of. Des Moines ten years, from 1891 to 1901 .

Chloe Funk-Wife of V. P. 'Twombly.

W. W. Baldwin-Soldier and lawyer, proninent citizen of Burlington and president of its library association, became land commissioner of the C., B. \& Q. R. R. in about 1879, still in the employ of that company as vice-president and is an able writer on railroad questions.

John Burns-Soldier four years, sergeant Third Cavalry: afterward treasurer Van Buren county and postmaster at Keosauqua.

B. F. Elbert-Banker and member of Iowa Legislature.

Felix 'T. Hughes--Soldier, school teacher in Memphis, Mo., three years, lawyer in Lancaster, Mo., in 1880 removed to Keokuk, Towa, as general solicitor of the M., I. \& N. R. R., five years later president and attorney for the same road reorganized as the Keokuk and Western until it was sold to the C., B. \& $Q$., since which he has been local attorney for the C., B. \& Q. Meantime he has been mayor of Keokuk two years and judge of that city's superior court three years.

Ben Johnston-Soldier four years in Union army, promoted lieutenant in colored regiment, lawyer, county attorney, and died while United States consul in a Houduras port. 
B. F. Kauftman-Lawyer, and by many thought to be the foremost attorney in Des Moines when he died in the prime of life.

Rutledge Lea-Said to have been the best declaimer in the school, became an able lawyer but died when about forty years old.

Alvin J. McCrary-Soldier, lawyer, president Iowa State Bar Association, appointed by President Roosevelt a delegate to the congress of lawyers at the St. Lonis Exposition, and since 1900 has been attormey for two corporate companies at Binghampton, N. Y.

Craig L. Wright-Son of Judge George G. Wright and for many years an able lawyer in Sioux City.

Sam: M. Clark-Editor of the Gate City and member of congress.

J. H. Watts-First lieutenant in Third Cavalry and killed in battle.

Charles Leach-First lieutenant in Third Cavalry.

William C. Stidger-Soldier four years, second lientenant and adjutant of Fifteenth Iowa.

George Stidger-SSoldier and physician.

Addie Stidger-Wife of George C. Duffield.

John Baker-Soldier and physician.

William C. Harper-Lientenant in the Second Iowa, was killed at Ft. Donelson. .

W. H.Andrews, Irene Anderson, Lavina R. Baldwin, Rachel; Berger, Mary Bonney, John Bonner, Jerome Briggs and two sisters, Miles Burns, Mary Claflin, Isou Canaja, Eliza Day, Henry Easling, Susanna Fellows, Lutie Ganes, Lizzie and Susie Farrison, Ellen Brewster, Clarissa Hartson, Samuel and Benjamin Hearn, Thomas and Orra Henry, Sally Jordan, Stanslow Julien, Christopher Kauffman, Augusta Kinnersly, Lemuel Kincade, Lena Lea, Anna and Will Manning, Josie Manguin, Flavius, Scott and Susan Miller, Hattie McArthur, Nelson McCrary, William MeBride, Sarah and Vina Morris, Elizabeth Myers, Frances Miller, Lida Moore, Emma and Amandus Pearson, Henry Potter, Mary Purnell, James and Mary Rankin, Laura Rowley, Lewis Rye, John C. Smith, Melissa Stannard, Fletcher and Mary St. John, Clarence and 
Amanda Walker, Thomas Thornburg, Peter Watts, Saralı Warren, Mary Wheelan, Solon Wilson, Dora and Mary Wright, Samuel Hogne, Elizabeth Marshall.

The building in which Mr. Lane tanght his schools and the church he built in Keosauqua have long since been torn down that modern ones might take their places, and Mr. Lane has been dead nearly twenty-six years; but his memory is still cherished in the town, and will be after those who knew and loved him in the flesh are dead and gone. In the present Congregationalist church on the wall, above and iback of the pulpit, there hangs a large and life-like picture of Mr. Lane, who seems to be-looking over the congregation, his eyes fondly resting upon the beautiful front window, which bears this inscription: "Daniel Lane, D. D., of the 'Iowa Band,' and the first pastor of this church. By his students, testifying their affection and esteem, and commemorating his work and character as a Christian teacher." At the dedication of this chureh, in 1888, Thomas S. Wright delivered an appropriate and able address in behalf of Mr. Liane's students, some of whom had journeyed far to be present on the occasion. The foregoing narrative is a more able and just tribute to the character of Mr. Lane than I can personally offer in another form. But to show further how he was and still is regarded and appreciated by his former students, I here quote tributes which a few of them sent me at my request. Mrs. Rustin says: "I think Mr. Lane's suceess as a teacher was the result of his splendid scholarship and ability to impart knowledge, to his kind, wise and firm control of his scholars, and to his broad views and aims. He was much more than a mere pedagogue, with an eye upon his pecuniary reward. He had a great longing for the personal profit of his scholars, and through them for the future welfare of Iowa. As I think of it now, he must have taken up teaching in Keosauqua as a necessary corollary of his ministerial work, his conception of the work of a home missionary was that broad. He saw that the hope of Iowa lay in the morality and intelligence of her citizens. As a minister, and as a citizen himself he obeyed the call to do what he could to mold the minds and characters of the rising generation. And, Oh, the personality of the man! Sincerity radiated from his 
countenance. Even a look from his honest, blue eyes blessed the one on whom it rested."

By Alvin J. MeCrary: "No man can fully estimate a life so poured out on his pupils as was Mr. Lane's. In lasting influence never did a teacher more surely fasten his wise thoughts upon his scholars. And he personally followed them in after years with his loving counsel. He was one of the few who could talk of Divine things without cant. He was truly the friend of youth, yet you could not think of him as ever having had any youth. Man's value to man is the true measure of greatness. But by that standard Daniel Lane's greatness will never be realized in this world."

By Judge Felix T. Hughes: "We loved Professor Lane very dearly, and I have always regarded him as a wonderful educator, and in other respects a really wonderful man. His christain virtues and his anxiety for the advancement of his scholars were really admirable, and no thoughtful young man or woman could work for other than his highest esteem. 1. can see him now before the classes, his face aglow' with interest and anxiety for the success of his pupils. He was so tender, so patient, and yet so firm that he never let a pupil go until he understood just what the lesson was intended to teach. He was so perfectly informed himself that it seemed a delight to him to exert himself in the interest of the subject under consideration, and he was so free in the use of simple and plain language that it all seemed real eloquence, and held us to the closest attention."'

By. V. P. Twombly: "Mr. Lane was loved by all his scholars. He was stern on occasion, but very just. As an example of his thoroughness I may relate that our arithmetic classes seemed to have trouble to remember, ' 5280 feet make a mile', that sentence was written at the top of the blackboard, and kept there until it was impressed upon their memories. And I venture that few, if any, of those scholars, if asked today, would fail to answer promptly, '5280 feet make a mile.' Mr. Lane was a strong, earnest, christian character; not a great preacher, but one who truly exemplified the Christ-life in his daily walk and conversation. He certainly was a great teacher and leader of young people." 
And Mrs. Twombly says: "My strongest impressions of Mr. Lane in. the school room were made by his quiet talks before or after reading a Scripture lesson and praying, every morning, on opening the school; and his repeating over and over again 'The fruit of the Spirit is love, joy, peace, long-suffering, kindness, goodness, faithfulness, meekness; against such there is no law." (Gal. V. 22-23.)

By W. W. Baldwin: "Mrr. Lane was more than a teacher and preacher. He was a great example. His precepts were sound, but his life was more than all his precepts. He was not simply an upright and pure-minded man, but was the embodiment of uprightness and high character. T think that he combined in himself more nearly the best type of a patriotic citizen, the faithful pastor, and the conscientions teacher than any man with whom my life has been associated. This explains his influence upon his pupils, an influence in the formation of character and habits above any influence of maxims or books, and one which endures in our minds and lives even now after the lapse of more than half a century, not only as a blessed memory but also as a vital force."

In 1862 another protracted illness prevented $\mathrm{Mr}$. Lune from teaching the final term, eleven weeks of his second school. That probably convinced him that he was no longer equal to the strain of continuous teaching. At any rate as soon as he was able he returned to active work in the ministry, in which he served as pastor at Eddyville four years, and at Pleasant Plains six years; he then retired from pastoral work on account of impaired hearing. In 1872-73 he assisted in raising funds for Iowa College. He then moved to Oskaloosa, chiefly to enjoy the fellowship of "Father" Turner, who in age and feebleness lived there with his daughter. Mr. Lane still preached at times, and for short periods undertook pastoral charge of churches which were temporarily without installed pastors. In that capacity he was again at Eddyville six months, and three months at Keosauqua. While in Iowa he was pastor of churches twenty-one years, a teacher eleven years and college agent two years; making in all thirty-four years of active labor, including the two years when he was both pastor and 
teacher in Keosaukua. And in the meantime he was a trustee of Iowa College for twenty-six years.

As old age crept upon them Mr. and Mrs. Lane yearned for the land of their youth, and for their relatives and friends who still lived there. So they left Iowa and went back to Maine in December; 1882. In. order to be near Mrs. Lane's relatives they bought. a small, rural home about a mile from the little village of Freeport. It will please his Iowa friends and pupils to learn that the generous and self-denying Daniel. Lane had enough means to supply his moderate wants in the evening of life. Mrs. Lane's sister, Miss Anna Staples, writes . me, "One of Mr. Ixane's Iowa friends advised him to invest some money in land, so that he would have something for old age, or to leave to his wife if she survived him, which she did for ten years. 'The investment proved to be a good one so when he came here he was able to buy a small place and live very comfortably. After he died some of his money was lost through his agent in the west; but there was enough left to last Mrs. Lane through, and what there was cver was to go to Towa College and the missionary societies. He was to the last a cheerful giver. and when he received a gift he would give it to some good cause instead of using it for his own benefit."

Mi. Lane lived a little over seven years after returning to Maine, and died April 3,1890, at the age of seventy-seven years and twenty-three days. Of his closing years Miss Staples writes, "His last days were passed quietly in reading and study, cultivating his garden and preaching occasionally. IIe was a constant attendant.at church and mid-week prayer meetings. He had a larye Bible class of men and women in the Sunday school, and a neighborhood prayer meeting at his home on Saturday evenings."

Thus, contrary to the dark prophecy of the physician in his academy days, although seriously handicapped by a frail body and frequent illness, this good man labored long and successfully for God and humanity, and "came to his grave in a full age, like as a shock of corn cometh in its season." 
Copyright of Annals of Iowa is the property of State of Iowa, by \& through the State Historical Society of Iowa and its content may not be copied or emailed to multiple sites or posted to a listserv without the copyright holder's express written permission. However, users may print, download, or email articles for individual use. 